\section{Megagauss Field Available for Research}

\section{F. Herlach, Leuven}

It is virtually impossible to generate a magnetic field of more than $100 \mathrm{~T}(=1$ megagauss) in the laboratory for a time interval of more than a few microseconds. This is due to the high energy density $\left(B^{2} / 2 \mu_{\circ}=40 \mathrm{~kJ} / \mathrm{cm}^{3}\right.$ at $\left.100 \mathrm{~T}\right)$ and the related magnetic stress 140 $\mathrm{GPa}$ ). Maraging steel which is one of the strongest construction materials, has a yield strength of $2 \mathrm{GPa}$. The energy density of gasoline is $36 \mathrm{~kJ} / \mathrm{cm}^{3}$, and that of high explosives is even lower $1 \approx 10$ $\mathrm{kJ} / \mathrm{cm}^{3}$ ) because these contain the oxygen needed for combustion.

To generate a strong magnetic field, magnetic energy must be transferred to a volume where it is confined by currents in a conducting material. As the ultrastrong field pushes the conducting wall away, the energy transfer must be fast; this results in a skin effect which concentrates the magnetic energy at the conductor surface facing the magnetic field. Under the conditions of adiabatic heating and a well developed skin effect there is approximate equipartition between the power that goes into the magnetic field and into Joule heating. For copper, this results in a temperature increase of $\approx 1200 \mathrm{deg} / \mathrm{MG}^{2}$; the boiling point is reached at $150 \mathrm{~T}$.

Nevertheless, it is not this effect which ultimately sets a limit to the field that can be generated with a given apparatus, characterized mainly by the power input to the field volume. Because of the skin effect, the magnetic stress is applied to the material at the surface, and the necessary fast rise of the field pulse results in a shock wave being driven into the material. Shock waves are governed by fairly simple laws and it is a straightforward matter to calculate the speed at which the surface of the conductor recedes from the magnetic field. This is well approximated by the particle speed behind the shock wave which is given by a semi-empirical equation of state of the material as a function of the pressure. This speed is approximately proportional to $B^{3 / 2}$; for copper this is $1.7 \mathrm{~km} / \mathrm{s}$ at $500 \mathrm{~T}$.

Generating a megagauss field is thus mainly a matter of speed; the most direct method is the compression of magnetic flux in a conducting cylinder imploding at very high speed. The cylinder may be driven by means of high explosives (as first published by C.M. Fowler et al. in 1960: J. Appl. Phys. 31 588) or by electromagnetic forces (1966: E.C. Cnare, J. Appl. Phys. 37 3812).

It is evident that experimentation with megagauss fields requires the development of drastically novel one-shot experimental techniques. As destruction is inherent in the magnetic field itself, this is accepted as a matter of fact and experiments are designed to yield a maximum of information from each single shot, and to minimize the damage to the experimental setup. The expendable parts of the setup must thus be inexpensive as well as most reliable. For magnetic fields up to $\sim 300 \mathrm{~T}$, the simple technique of directly discharging a very fast capacitor bank into a small single turn coil has been developed into a practical laboratory instrument (1973: F. Herlach and R. McBroom, J. Phys. E 6 652; 1985: K. Nakao et al., J. Phys. $E$ in press). This technique is most energy efficient and therefore produces the smallest damage possible. The tiny coils (typical volume $\sim 1 \mathrm{~cm}^{3}$ ) explode with great violence but the sample space is not much disturbed because in this arrangement the fragments are pushed away from the sample by the magnetic field. With little additional protection against the after effects of the blast associated with the coil explosion, even delicate samples can survive in an exploding single turn coil.

Megagauss fields are now available for experimentation at a few laborato- ries: the Los Alamos Scientific Laboratory (explosive-driven flux compression in cylindrical and plane geometry), the Kurchatov Institute of Atomic Energy (10 MG reproducibly with explosives, single turn coil), the Institute for Solid State Physics at the University of Tokyo (electromagnetically driven flux compression, single turn coil), the Institute of Hydrodynamics, Novosibirsk (explosivedriven flux compression) and Leningrad Polytechnical Institute (single turn coil). At Los Alamos, the Kurchatov Institute and Tokyo, the first solid state experiments have been carried out, mostly with optical methods in the visible and infrared part of the spectrum (reviewed by N. Miura and F. Herlach in Strong and Ultrastrong Magnetic Fields, Springer Topics in Applied Physics, Vol. 57, 1985). Conferences on the generation and applicaiton of megagauss fields were held in 1965 (Frascati), 1979 (Washington, D.C.) and 1983 (Novosibirsk). The next conference will be held at Santa Fe, N. Mex. from 14 to 17 July 1986.

Now that megagauss fields are available under conditions suitable for experiments, it is intended to stimulate interest in experimenting with them in solid state physics and in other areas of pure and applied physics. To this end we should like to invite our colleagues to present proposals at the July conference for experiments that might be of interest. Information regarding the conference is available from: C.M. Fowler, mail stop J970, Los Alamos Scientific Laboratory, Los Alamos, N. Mex. 87545 or from F. Herlach, Katholieke Universiteit Leuven, Celestijnenlaan 200 D, B-3030 Leuven (Belgium).

\title{
TISTITUTO TAZIONALE DI FISICA NUCLEARE \\ ISTITUTO NAZIONALE DI FISICA NUCLEARE Laboratori Nazionali di Legnaro

TWO POST-DOCTORAL FELLOWSHIPS
IN EXPERIMENTAL NUCLEAR STRUCTURE PHYSICS

Applications are invited for the above posts which are now vacant and are funded by the I.N.F.N. for the period 1 October 1986 - 30 September 1987. The fellowships are open onIy to non-italian citizens. Deadline for applications 1 April 1986. The successful candidates will be expected to initiate and assist with research at the XTU tandem van de Graaff, which is now operational at the Laboratori Nazionali di Legnaro.

Applicants are expected to hold a Ph.D. degree in nuclear structure physics and should have an aptitude for pursuing research in experimental physics. The salary will be 24000000.LIT gross per annum corresponding to 1600000 . - Italian Lire per month net, plus travel expenses from home institution to Legnaro and return.

Applications with full C.V. and names of two referees and possibly an introduction letter from a senior scientist to:

Prof. Cosimo Signorini, Director

Laboratori Nazionali di Legnaro - I.N.F.N.

V. Romea, 4, 1 - 35020 Legnaro (Padova) Italy

(Phone: (49) 6412 00; Telex: 430384 LNL I) 